\title{
The Analysis of Hierarchical Structure of Mesoporous Silica in Nanometer Scale by Small Angle Scattering Method
}

\author{
S. Hadi ${ }^{1}$, S. Suryajaya ${ }^{1}$, I. Wijaya ${ }^{2}$, A. Rahmandari ${ }^{2}$, M.C. Prihatiningsih ${ }^{2}$, \\ I. Prasetyo ${ }^{3}$, A. Patriati ${ }^{4}$, E.G.R. Putra ${ }^{2,4, *}$, S. Soontaranon ${ }^{5}$ \\ ${ }^{I}$ Department of Physics, Lambung Mangkurat University, Banjarbaru, Jl. A. Yani Street, KM. 36, Banjarbaru, \\ South Kalimantan, Indonesia \\ ${ }^{2}$ Polytechnic Institute of Nuclear Technology, National Nuclear Energy Agency, \\ Jl. Babarsari Yogyakarta 55281, Indonesia \\ ${ }^{3}$ Department of Chemical Engineering, Gadjah Mada University, Jl. Grafika No. 2, Yogyakarta 55281, Indonesia \\ ${ }^{4}$ Center for Science and Technology of Advanced Materials, National Nuclear Energy Agency, \\ Puspiptek Area, Serpong, Tangerang 15310, Indonesia \\ ${ }^{5}$ Synchrotron Light Research Institute (SLRI), University Avenue, Muang District, Nakhon Ratchasima 30000, Thailand
}

\section{ARTICLE INFO}

\section{Article history:}

Received 16 November 2016

Received in revised form 7 September 2017

Accepted 10 September 2017

\section{Keywords:}

Mesoporous

Small angle scattering

Nanostructure

Hierarchical structure

\begin{abstract}
A B S T R A C T
The analysis of hierarchical structure of mesoporous silica material with template of cetyltrimethylammonium bromide (CTAB) with co-surfactants of tetramethylammonium hydroxide (TMAOH) and Triton X-100 was conducted by using the technique of small angle scattering (SAS) using neutron (SANS) and X-ray (SAXS). The analysis was supported by the data of nitrogen absorption and electron microscopy. The analysis showed that the concentration of CTAB affected the characteristics and pore structures of particles. The increase of co-surfactant concentrations tended to form particles that were more uniform in size and more regular in the shape of the sphere. The results of SAS analysis showed that the morphology, shape, and size of the large particles were arranged by smaller (primary or secondary) particles that had pores. The variation of surfactant templates had influenced the formation of pore structure. For CTAB-TMAOH, it had resembled MCM-41 type which has a hexagonal structure, whereas for CTABTriton X-100, it would have resembled MCM-48 type which has a cubic structure. The particles that have a high surface area which resembled pore structure MCM-41 has been able to set up by using $0.25 \mathrm{M}$ of CTAB with $0.040 \mathrm{M}$ of TMAOH. Moreover, the particles which resembled pore structure MCM-48 were able to set up by using $0.03 \mathrm{M}$ of Triton X-100 with $0.4 \mathrm{M}$ of CTAB. The analysis of SANS data that was supported by electron microscopy results is entirely showing a complete information of the particles formed by each template. Whereas SAXS analysis that supported by nitrogen adsorption method is fully confirming the information of pore characteristics.
\end{abstract}

\section{INTRODUCTION}

Mesoporous silica materials have been used in many applications, such as medium separator, absorber [1], catalyst [2], and carrier for drug delivery $[2,3]$. Several methods have been applied to

*Corresponding author.

E-mail address: giri@batan.go.id

DOI: https://doi.org/10.17146/aij.2018.848 synthesize mesoporous silica materials to obtain a tuneable and controllable pore structures or highly ordered pore structures [4,5] such as by using conventional sol-gel processes, liquid crystal templating method, or by nanocasting strategy [5].

To obtain such information as the mechanism of formation of the mesoporous silica structure, characterization methods that can be used to observe the structural changes during the formation of 
mesoporous silica materials are certainly needed. Several methods of characterization such as X-ray diffraction [4], small angle scattering (SAS) of neutron or X-ray [6-8], electron microscopy [9], and transmission electron micrographs [10], can be used for this purpose. The structure of the material could be observed using diffraction method whereas surface morphology could be observed using electron microscopy method. However, the hierarchical structure of mesostructured silica materials could not be clearly obtained by electron microscopy, X-ray diffraction, microcalorimetry, or nitrogen absorption surface area analyzer methods. Small angle scattering of neutron (SANS) or X-ray (SAXS), can be simply employed as characterization method to analyze the hierarchical structure of mesostructured silica materials. Furthermore, these methods can be used to obtain some information of the mechanism of formation of the mesoporous silica structure in nanometer scale.

In the mesoporous silica research, the determination of the shape and size of secondary particles as well as the primary particles and pores structure and size at the same time is highly desirable to elucidate the intrinsic properties of the materials. SAS analysis has been proofed as a method to obtain the detail information about the characteristics of mesoporous silica material in the nanometer size range. Until now, SAS analysis could clarify the formation of globular sphere and worm-like shapes in polyoxyethylenefluoroalkyl ether templates [6], changes of mesoporous structure caused by salt on some sources of silica [7], and the particle size of the ionic surfactants [9]. However, several methods of materials characterization such as microscopy could not noticeably represent an exhaustive analysis about the bulk structural characteristics in nanometer scale. Further analysis of the nanostructure of mesoporous silica using SAS method including data processing software analysis, producing a calculation model structure, is very important to provide more information of the material at nanometer-scale level.

In this work, the combined data of SANS and SAXS will be processed and fitted with global fitting calculation model. These SAS data are also confirmed to data of the electron microscopy (EM) and the BET (Brunauer-Emmett Teller) method of analysis of gas adsorption data for constructing the nanostructure of mesoporous silica. The study is suitable to investigate the hierarchy of nanostructure of mesoporous silica material such as the information of secondary particles, primary, subparticles, pores and pore framework. Those properties were believed as a constituent of mesoporous silica material.
The variation of CTAB-TMAOH and CTABTriton X-100 surfactant concentration which had special characteristics in forming mesoporous silica material would also be investigated. The results of this research would potentially provide more detail information to fabricate the mesoporous silica materials needed or for specific applications.

\section{EXPERIMENTAL METHODS}

\section{Materials}

The materials used consisted of tetraethyl orthosilicate (TEOS) as a source of $\mathrm{SiO}_{2}$, cetyltrimethylammonium bromide (CTAB), tetramethylammonium hydroxide (TMAOH), Triton $\mathrm{X}-100$, Aquades, $\mathrm{NaOH} 1 \mathrm{~N}$, Ethanol $97 \%$, and $\mathrm{HCl}(1 \%$ and $0.1 \mathrm{~N})$. All of those chemicals were purchased from Merck without any further purifications.

\section{Synthesis}

The research was conducted on mesoporous silica material that had been produced by bottom-up method. It had been synthesized as follows: samples with ratio $1.0 \mathrm{SiO}_{2}: x$ CTAB $: y$ TMAOH $: 0.15$ $\mathrm{NaOH}: 58 \mathrm{H}_{2} \mathrm{O}(x-y$ based on sample 1,2 and 3) and $1.0 \mathrm{SiO}_{2}: x$ Triton X-100 $: y \mathrm{CTAB}: 0.5 \mathrm{NaOH}$ : $58 \mathrm{H}_{2} \mathrm{O}(x-y$ based on samples 4,5 and 6$)$ as listed in Table 1. TEOS was added at a temperature of $35-40{ }^{\circ} \mathrm{C}$, and the mixture was stirred for an hour. Afterward, the mixture was aged at temperature of 100-130 ${ }^{\circ} \mathrm{C}$ for five days. The precipitation was conditioned at $\mathrm{pH}=10$ for $3-5$ days with the addition of $1 \% \mathrm{HCl}$. Then, the precipitate was filtered and washed with demineralized water + alcohol + $\left(\mathrm{HNO}_{3} 1 \mathrm{~N}\right.$ for samples 1,2 and 3$)$ or $(\mathrm{HCl} 1$ $\mathrm{N}$ for samples 4, 5 and 6) with ratio 90: 9: 1. The precipitate was dried at $90-100{ }^{\circ} \mathrm{C}$ for one day and then calcined at temperature of $550{ }^{\circ} \mathrm{C}$ for five hours. Table 1 shows the composition of the template combinations of prepared for samples that were characterized in this study.

Table 1. The composition of the template combinations for the preparation of mesoporous silica materials

\begin{tabular}{cl}
\hline Sample No. & \multicolumn{1}{c}{ Concentration of template } \\
\hline 1 & $0.25 \mathrm{M} \mathrm{CTAB}+0.015 \mathrm{M} \mathrm{TMAOH}$ \\
2 & $0.25 \mathrm{M} \mathrm{CTAB}+0.030 \mathrm{M} \mathrm{TMAOH}$ \\
3 & $0.25 \mathrm{M} \mathrm{CTAB}+0.040 \mathrm{M} \mathrm{TMAOH}$ \\
4 & $0.03 \mathrm{M}$ Triton X-100+0.25 M CTAB \\
5 & $0.03 \mathrm{M}$ Triton X-100+0.30 M CTAB \\
6 & $0.03 \mathrm{M}$ Triton X-100+0.40 M CTAB \\
\hline
\end{tabular}

These prepared combinations were then further characterized by SAS method in order to obtain the structural changes of the mesoporous silica in nanometer-scale level. 


\section{Characterization}

The characterization technique used were SANS and SAXS. SANS experiments were performed using a 36-meter SANS spectrometer at the National Nuclear Energy Agency (BATAN) of Indonesia, where as SAXS experiments were conducted using a synchrotron SAXS facility at the Synchrotron Light Research Institute (SLRI) in Thailand. The SANS experiments were setup to cover the medium to low momentum transfer $q$ range. Meanwhile, the medium to high $q$ range was setup in the SAXS experiments.

The analysis result of scattering data was then examined with additional data from EMS result and nitrogen adsorption method in obtaining the morphology, size and structures of the particles and pores.

\section{Analysis}

A fitting calculation model was completed using a software SAS fit through a global fitting mode. A global fitting calculation model was used to analyze all scattering patterns covering low to medium momentum transfer $q$ range of SANS data, where as the scattering patterns of SAXS was analyzed for the Bragg peak in diffraction angle $2 \theta$ conversion in the high $q$ range.

A spherical shape model was used to analyze the results of SANS characterization, using lognormal function as size and its distribution of particle shape, and peak calculation model based on the Gaussian function as pore structure factor and mass fractal with Gaussian cut-off functions as the particle structure factor, secondary particles (Fig. 1).

The equation of fitting calculation model used is presented as follows:

\section{Size and shape distributions:}

\section{Sphere (lognormal)}

$\operatorname{LogNorm}(X, \mu, \sigma, p)=\frac{\mathrm{N}}{\mathrm{C}_{\mathrm{LN}}} \frac{1}{\mathrm{X}^{\mathrm{P}}} \exp \left(-\frac{\ln \left(\frac{X}{\mu}\right)^{\frac{2}{2}}}{2 \sigma^{2}}\right)$

with:

$$
\mathrm{C}_{\mathrm{LN}}=\sqrt{2 \pi} \sigma \mu^{1-\mathrm{p}}\left((1-\mathrm{p})^{2} \frac{\sigma^{2}}{2}\right)
$$

where $\mathrm{N}$ is the scale scattering, $\sigma$ is the degree of particle size polydispersity, and $\mu$ provides information on particle size.

\section{Structure factor}

\section{Peak (Gaussian amplitude)}

$$
y\left(x, a, x_{c}, \sigma, c_{0}\right)=a \exp \left[-\frac{1}{2}\left(\frac{x-\mathrm{x}_{\mathrm{c}}}{|\sigma|}\right)^{2}\right]+\mathrm{c}_{0}
$$

with $a$ is the amplitude, $\mathrm{x}_{\mathrm{c}}$ is the position of amplitude peak, $\sigma$ is the scale parameter of width peak, and $\mathrm{c}_{0}$ is the background of scattering.

\section{Mass fractal (Gaussian cut-off)}

$$
h_{\text {Gauss }}(\mathrm{r}, \xi)=\exp \left[-\left(\frac{\mathrm{r}}{\xi}\right)^{2}\right]
$$

Where $r$ is the scattering characteristic of a single particle (primary particle size), and $\xi$ is a measure of fractal aggregation, with additional information from the fractal dimension.

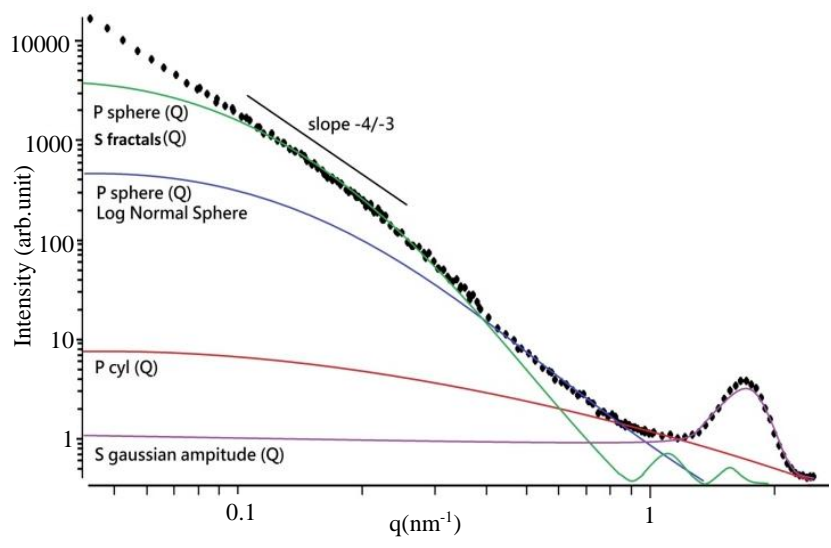

Fig. 1. The calculation models of several combined form and structure factors applied on a SANS scattering profile.

\section{RESULTS AND DISCUSSION}

\section{Surface area and pore size}

The pore characteristics were examined using nitrogen adsorption method and are given in Table 2.

Table 2. Adsorption of nitrogen characterization results

\begin{tabular}{ccc}
\hline Sample & Area $\left(\mathrm{m}^{2} / \mathrm{g}\right)$ & Porediameter $(\mathrm{nm})$ \\
\hline 1 & 628.1 & 3.804 \\
2 & 640.7 & 3.768 \\
3 & 623.5 & 4.147 \\
4 & 653.7 & 3.481 \\
5 & 829.2 & 3.123 \\
6 & 873.5 & 2.929 \\
\hline
\end{tabular}

The characterization of nitrogen adsorption provides the information about the surface area and pore diameter of the material. The result shows that the increase of co-surfactant concentration on 
samples 1-3 in general did not significantly change the pore diameter and surface area, whereas on samples 4-6, CTAB as co-surfactant significantly decreased the pore diameter or increased the surface area. The ability of the strong hydrophobic chains of CTAB to interact with the hydrophobic domain of Triton X-100 significantly reduced the size of the micelles in the solution that formed the pores. Therefore, it can be confirmed that the pore size has an effect on the surface area wherea smaller pore size will result in more poresor larger surface area. This was previously difficult to observe by electron micrograph [10].

\section{Morphology and characteristics of particles (EM)}

Figure 2 shows the electron micrograph of the morphology of mesoporous silica samples. A large particles, about $200 \mathrm{~nm}$ and more, has been obtained from each sample. However, in general,
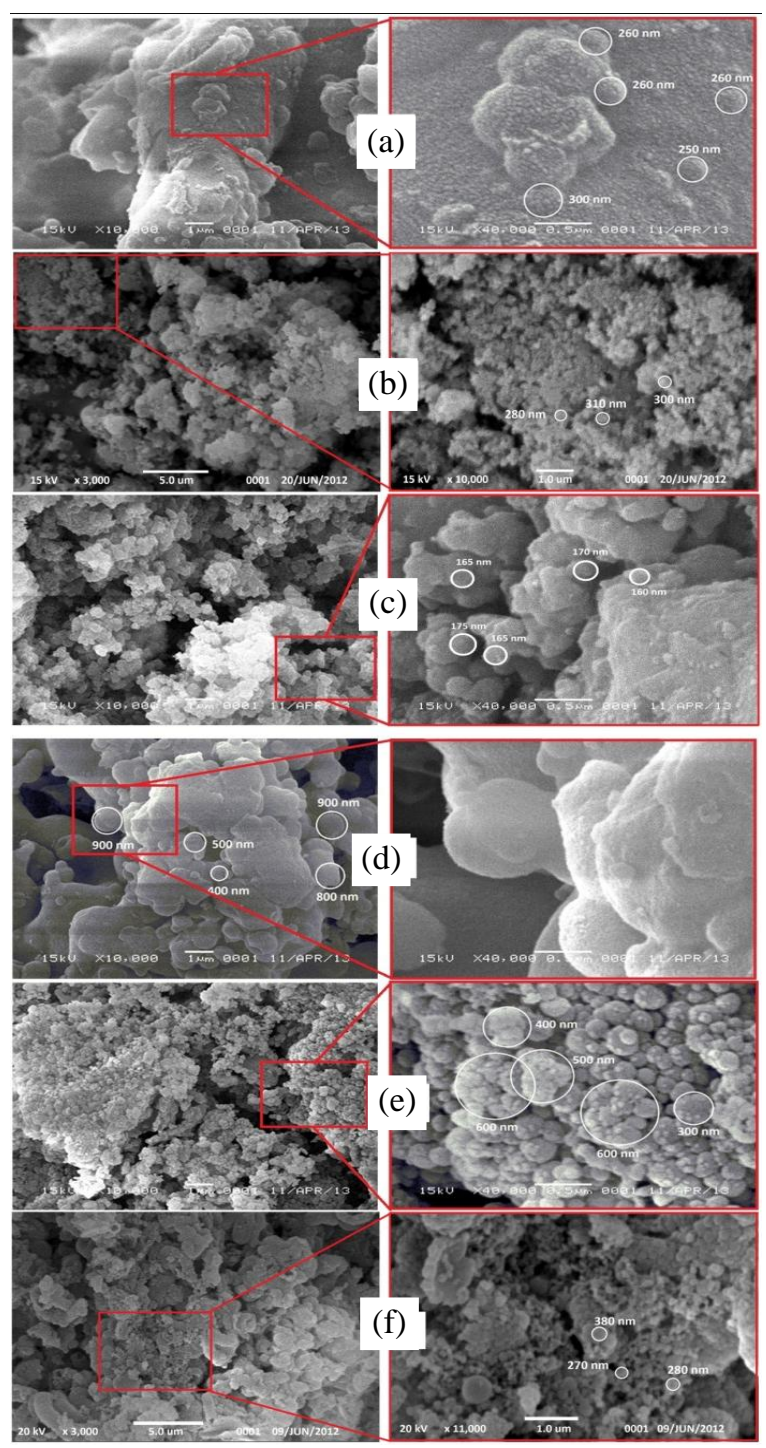

Fig. 2. Electron micrograph of mesoporous silica (a) Sample 1 (b) Sample 2, (c) Sample 3, (d) Sample 4, (e) Sample 5, and (f) Sample 6. two different morphologies are observed from the samples. A non-aggregated form is mostly found in samples 1-3, Figs. 2(a)-(c), whereas an aggregated form with a large particle size and composed by smaller particles is found in samples 4-6, Figs. 2(d) -(f). The range of particle sizes that was obtained from the result of electron microscopy characterization could be employed to support SAS analysis.

\section{Particle size and distribution (SANS)}

The size of particles formed by the variations of CTAB concentration as a template could be determined through a fitting calculation model as illustrated in Fig. 1, on SANS data that is shown on Figs. 3 and 4.

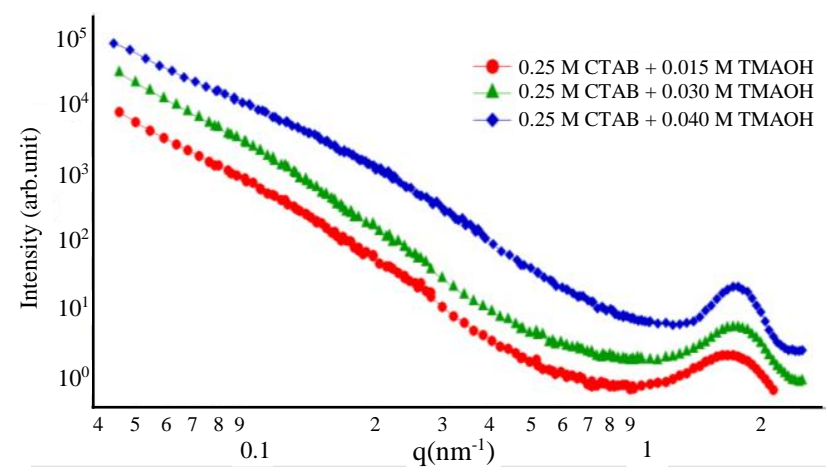

Fig. 3. SANS scattering curves of $0.25 \mathrm{M}$ CTAB with the addition of TMAOH concentration.

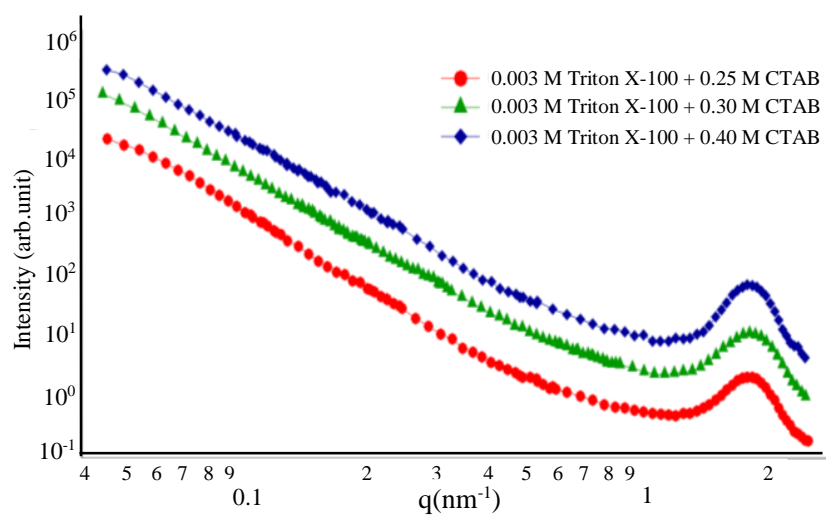

Fig. 4. SANS scattering curves of $0.03 \mathrm{M}$ Triton $\mathrm{X}-100$ with the addition of CTAB concentration.

A spherical shape model with log normal function was applied to analyze the SANS data in order to obtain the size and its distribution of the particles, i.e., the primary particles, while mass fractal with Gaussian cut-off functions was employed to determine the secondary (bulk) particles or fractal aggregation with additional 
information of the fractal dimension (eqs. 1-3). The fitting SANS data analysis is given in Table 3.

Table 3. The fitting result on SANS data of mesoporous silica

\begin{tabular}{cccc}
\hline $\begin{array}{c}\text { Sample } \\
\text { No. }\end{array}$ & $\begin{array}{c}\text { Average } \\
\text { diameter of sub } \\
\text { particle (nm) }\end{array}$ & $\begin{array}{c}\text { Average } \\
\text { diameter of a } \\
\text { particle (nm) }\end{array}$ & $\begin{array}{c}\text { Type of } \\
\text { particle }\end{array}$ \\
\hline 1 & 46.6 & 263.6 & Primary \\
2 & 51.3 & 317.2 & Primary \\
3 & 22.3 & 174.7 & Primary \\
4 & 34.9 & 494.8 & Secondary \\
5 & 24.5 & 584.1 & Secondary \\
6 & 36.6 & 385.7 & Secondary \\
\hline
\end{tabular}

The information could be extracted from the fitting results that large particles (bulk) were definitely arranged from small particles and the template of $\mathrm{CTAB}+\mathrm{TMAOH}$ produced large particles with rough surface, while the template of CTAB + Triton X-100 produced the particles with smooth surface.

The change of TMAOH concentration in the samples No. 1, 2 and 3 (Fig. 3) showed that large particles which were formed were composed of smaller size particles. This is unlike the particles formed during the concentration change of CTAB, as showed by samples No. 4, 5 and 6 (Fig. 4), which were not composed of smaller particles. In general, the particles are identified as primary and secondary particles (showed in Table 3).

\section{Pore structures (SAXS)}

From SAXS scattering pattern, the curves showed the uniformity of particles increased corresponding to the increase of TMAOH concentration in $0.25 \mathrm{M}$ of CTAB (Fig. 5) and CTAB concentration in $0.03 \mathrm{M}$ of Triton X-100 (Fig. 6), where as the increasing of Bragg peak intensity followed by the emergence of other small Bragg peaks provides the information of pore regularity.

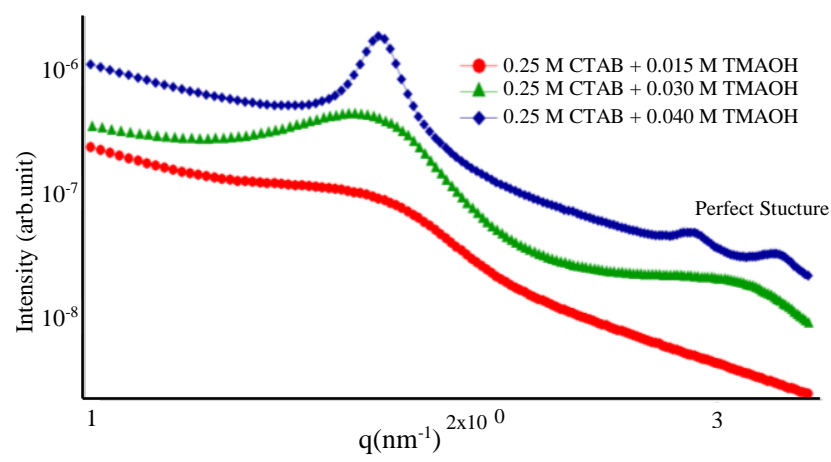

Fig. 5. SAXS scattering curves of $0.25 \mathrm{M}$ CTAB with the addition of TMAOH of three different concentrations.

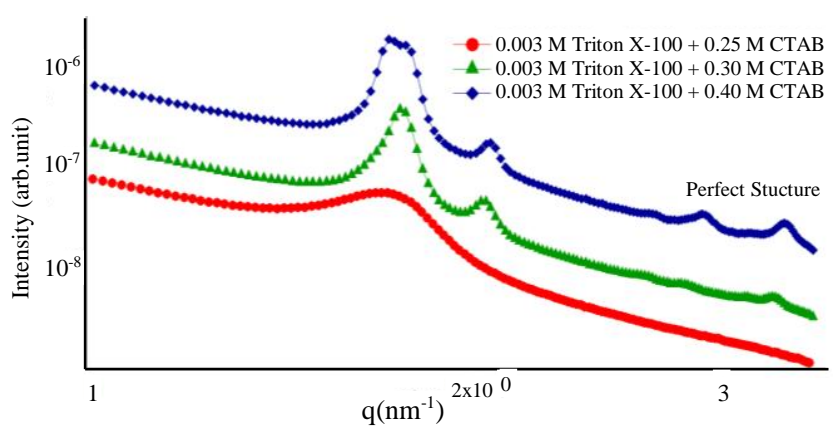

Fig. 6. SAXS scattering curves of $0.03 \mathrm{M}$ Triton X-100 with the addition of $\mathrm{CTAB}$ of three different concentrations.

The results of SAXS scattering at $q$ shows diffraction characteristics of the relationship:

$$
2 \theta=2 \operatorname{arc} \sin \frac{q \lambda}{4 \pi}
$$

with $2 \theta$ is the diffraction angle, $q$ is the scattering momentum, and $\lambda=1.542 \AA$.

So based on field diffraction and position of diffraction peak of the curves, the hexagonal pore structure (Fig. 7) was obtained for $0.25 \mathrm{M} \mathrm{CTAB}$ with addition of $0.04 \mathrm{M} \mathrm{TMAOH}$ and cubic pore structure (Fig. 8) for 0.03 M Triton X-100 with addition of $0.4 \mathrm{M} \mathrm{CTAB}$. Where as the parameter of peak position (20) obtained uniformity of the $d$-spacing values of the pore structures.

$$
\lambda=2 d \sin \theta
$$

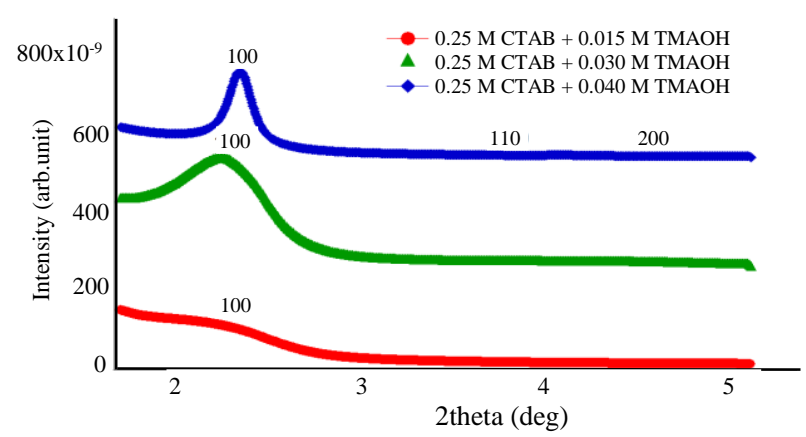

Fig. 7. Linear curve of SAXS profile $(2 \theta)$ on the condition $0.25 \mathrm{M}$ CTAB with the addition of TMAOH concentration.

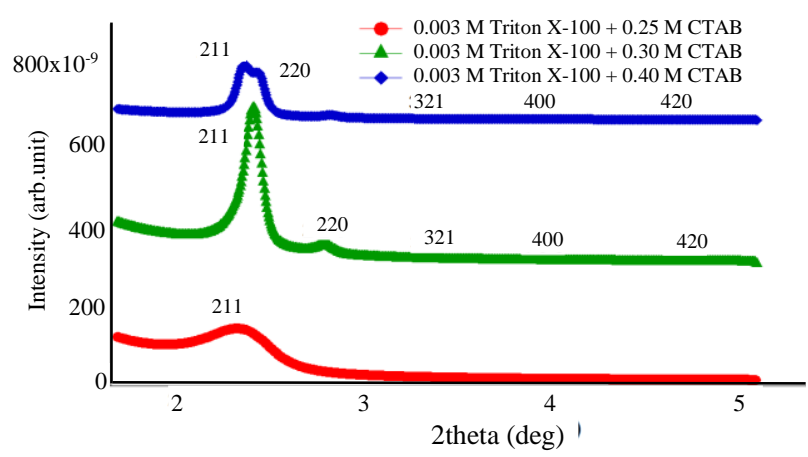

Fig. 8. Linear curve of SAXS profile $(2 \theta)$ on the condition $0.03 \mathrm{M}$ Triton X-100 with the addition of CTAB three different concentrations. 
Table 4. The extracted information of SAXS linear curve

\begin{tabular}{ccc}
\hline Sample & $2 \theta(\mathrm{deg})$ & $d$-spacing $(\mathrm{nm})$ \\
\hline 1 & 2.192 & 4.055 \\
2 & 2.251 & 3.950 \\
3 & 2.351 & 3.784 \\
4 & 2.340 & 3.802 \\
5 & 2.432 & 3.660 \\
6 & 2.383 & 3.734 \\
\hline
\end{tabular}

The analysis of SAXS linear curves, Figs. 7 10and 8 , found the peak position $(2 \theta)$ and $d$-spacing of pore structures $(\mathrm{nm})$, Table 4 . The increasing of each TMAOH concentration influences a larger Bragg diffraction peak shifted in $\mathrm{CTAB}+\mathrm{TMAOH}$ condition. Whereas the increasing of each CTAB concentration influences a smaller diffraction peak shifted in Triton X-100 + CTAB condition.

The information of pore size in terms of nitrogen adsorption characterization and $d$-spacing pore structure from SAXS linear curve analysis can be used to determine the thickness of pore frame. The proposed model is presented in Fig. 9.

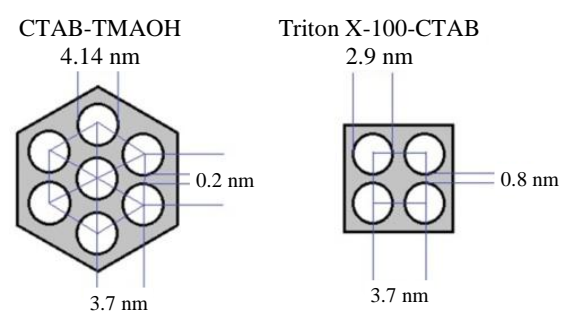

Fig. 9. Model of pore structures that formed on mesoporous silica material.

\section{Hierarchical nanostructures model}

The model showed the range of analyses that were performed using SAS and showed its ability to observe mesoporous silica nanostructure. The advantages of SANS in particles observation with smaller angles range of scattering, whichwas combined with SAXS which have range of scattering at larger angles, showed its capability to observe the formation of pore structure and uniformity that occured in the formed particles.

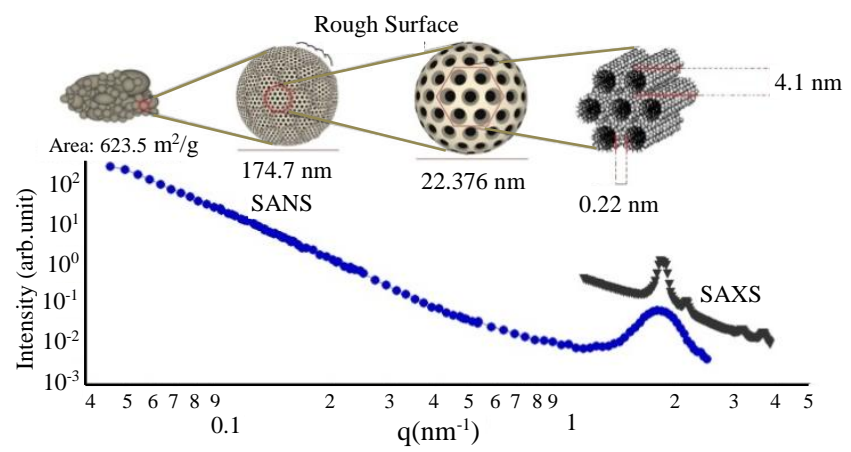

Fig. 10. Model of mesoporus silica CTAB-TMAOH surfactant (template).



Fig. 11. Model of mesoporous silica CTAB-Triton X-100 surfactant (template).

The models which are illustrated in Fig. 10 and Fig. 11 show a complete structural information about the particle pores, sub-particles, bulk particles, and the aggregations that certainly occured in the formation of mesoporous silica materials. Combinations of several methods of materials characterizations such as small angle scattering (SAS) of neutron or X-ray [6,7,9], electron microscopy [8], X-ray diffraction in low angle [4] and nitrogen adsorption have entirely demonstrated a powerful tool in obtaining the hierarchical structures of mesoporous materials.

\section{CONCLUSION}

The analysis of SANS data that was supported by electron microscopy precisely provided complete information on particles formed by each template, while SAXS analysis that was supported by nitrogen adsorption method provided complete information on those particles' pore structure characteristics. The template of $0.25 \mathrm{M}$ CTAB with the addition of $0.015 \mathrm{M}, 0.03 \mathrm{M}$, and $0.04 \mathrm{M}$ TMAOH showed the pore characteristics of hexagonal structure, whereas the template $0.03 \mathrm{M}$ Triton X-100 with the addition of $0.25 \mathrm{M}, 0.3 \mathrm{M}$, and $0.4 \mathrm{M} \mathrm{CTAB}$ showed the pore characteristics of cubic structure. The analysis also showed that the increase of particles uniformity corresponded to the increasing template concentration. The characteristics of CTAB + TMAOH particle is primary particle which has a rougher surface than the secondary particles on Triton $\mathrm{X}-100+$ CTAB template.

\section{ACKNOWLEDGMENT}

The authors would like to express their gratitude to Mr. Fenryco Pratama of Bandung Institute of Technology (ITB) for the sample preparation and data collection of the SAXS experiments. 


\section{REFERENCES}

1 A.Y.P. Sari, P. Taba and P. Budi, Ind. J. Chem. (2015) 249.

2 A. El-Toni, A. Khan, M. Ibrahim et al., Molecules 17 (2012) 13199. doi: 10.3390/molecules171113199

3 X. Huang, N. Young, H. Townley et al., Nanomater. and Nanotechnol. 4 (2014) 1. doi: $10.5772 / 58290$

4 H. Wanyika, E. Gatebe, P. Kioni et al., Afr. J. Pharm. and Pharmacol. 5 (2011) 2402. doi: 10.5897/AJPP11.592

5 J. Thielemann, F. Girgsdies, R. Schlögl et al., Beilstein J. Nanotechnol. 2 (2011) 110. doi: 10.3762/bjnano.2.13
6 F. Michaux, J. Blin, J. Teixeira et al., J. Phys. Chem. B. 116 (2012) 261. doi: 10.1021/jp209853r

7 P. Linton, A. Rennie and V. Alfredsson, Solid State Sci. 13 (2011) 793.

doi: 10.1016/j.solidstatesciences.2010.02.035

8 N. Hu, N. Borkar, D. Kohls et al., J. Membr. Sci. 379 (2011) 138.

doi: 10.1016/j.memsci.2011.05.053

9 S. Mascotto, D. Wallacher, A. Brandt et al., Langmuir 25 (2009) 12670. doi: 10.1021/la9013619

10 P. Linton, A. Rennie, M. Zackrisson et al., Langmuir 25 (2009) 4685.

doi: $10.1021 / 1 a 803543 \mathrm{z}$ 